\title{
Resistencias desde la periferia: población Rrom en España
}

\author{
José David Gutiérrez Sánchez
}

Universidad Pablo de Olavide

Resumen

El artículo analiza, a partir del caso de estudio de asentamientos chabolistas en Madrid y Barcelona, la población Rrom que ubicándose en la periferia de dichas ciudades malvive en condiciones de pobreza y exclusión social. Basándose en una investigación de corte cualitativo donde se aprecian similitudes en cuanto a los medios de subsistencia empleados por el colectivo, así cómo la movilidad constante tanto en España como en Europa, el artículo se interroga el papel que tienen las administraciones públicas a nivel local y regional en la búsqueda de soluciones que permitan una mayor inclusión social y mejoras en la calidad de vida. Mediante una comparativa de los asentamientos, esta investigación presenta la experiencia profesional desarrollada en asentamientos con altos niveles de exclusión social donde se pone de relieve, la necesidad de intervenir socialmente desde el Trabajo Social y además, bajo una armonización de protocolos de atención social a la población Rrom desde una perspectiva igualitaria dentro de la Unión Europea, debido sobre todo a las prácticas similares de subsistencia de la población y por compartir procesos de exclusión y pobreza desde origen.

Palabras clave:

Periferia; asentamientos; Rrom; gitanos; movilidad

Códigos JEL: 124, I28, I32, J15
RESISTANCE FROM THE PERIPHERY: RROM POPULATION IN SPAIN

\author{
Abstract
}

The article analyzes, based on the case study of shanty towns in Madrid and Barcelona, the Rrom population, which is located on the periphery of these cities, lives in conditions of poverty and social exclusion. Based on qualitative research where similarities can be seen in terms of livelihoods used by the collective, as well as constant mobility both in Spain and in Europe, the article questions the role of public administrations at local and regional level in the search for solutions that allow greater social inclusion and improvements in the quality of life. Through a comparison of the settlements, this research presents the professional experience developed in settlements with high levels of social exclusion where it is highlighted, the need to intervene socially from Social Work and also, under a harmonization of social care protocols to the Rrom population from an egalitarian perspective within the European Union, mainly due to the similar practices of subsistence of the population and for sharing processes of exclusion and poverty from origin.

Keywords

Periphery; settlements; Rrom; Gypsies; mobility

JEL codes: I24, 128, I32, J15

Fecha de recepción del original: 3 de diciembre de 2019; versión definitiva: 11 de diciembre de 2020 .

José David Gutiérrez Sánchez, Universidad Pablo de Olavide. Depamento de Trabajo Social y Servicios Sociales, Carretera de Utrera, Km, 1. Edificio 10.1.27. 41013- Sevilla-España.

E-mail:jdgutsan@upo.es. ORCID ID:0000-0003-0670-5426. 


\title{
Resistencias desde la periferia: población Rrom en España
}

\author{
José David Gutiérrez Sánchez
}

Universidad Pablo de Olavide

1. Introducción

El artículo presenta a la comunidad rumana y gitana procedente de Europa del Este, intentando ofrecer un análisis de la movilidad empleada por el colectivo en Europa hasta su asentamiento en España y concretamente en la periferia de grandes ciudades como son Madrid y Barcelona.

A la hora de abordar la definición de Rrom cabe en primer lugar realizar una puntualización dado que el propio término, en ocasiones, conlleva a interpretaciones diversas. Por un lado hay que destacar que Rrom se refiere concretamente a gitanos que, entre otros aspectos, hablan su propia lengua (romanés) y en este estudio además, son personas procedentes de Europa del Este. Por otro lado, la Unión Internacional Romaní adopta la doble erre con la intención de estandarizar y concretar la población a la que se hace referencia (Queraltó, 2005). La denominación es un tema a debate dado que numerosos autores e investigadores adoptan a su vez los términos řom/řomani o bien rromi para referirse como en este texto a los Rrom.

El Pueblo Gitano, según Vassilev (2004), constituye el mayor grupo étnico existente en toda Europa, donde encontramos una constelación de minorías que no son homogéneas desde una perspectiva histórica, cultural o religiosa (Baldoni, 2012; Dell'Agnese y Vitale, 2007; Magazzini y Piemontese, 2016). En la actualidad y en un contexto español marcado todavía por el impacto de la crisis económica iniciada en 2007 y que está padeciendo los efectos de la Covid-19, las intervenciones sociales orientadas a la inclusión social del colectivo Rrom y concretamente de Rumanía (donde se centra este trabajo ), han manifestado notoriamente la situación que este grupo poblacional exterioriza en cuanto su propia capacidad para insertarse en la sociedad y su vulnerabilidad a pesar de ser ciudadanos europeos de derecho. Dentro de esta situación, se establecen resistencias o espacios periféricos que a pesar de ser puntos estancos, presentan una movilidad interna bastante importante en cuanto a número de personas que proceden de Rumanía o bien de diversas partes de España o de Europa Occidental (Clave-Mercier y Olivera, 2018; Gutiérrez, 2017; Harper, Steger y Filcak, 2009).

De otra parte, el proceso de integración europea iniciado por la UE y Rumanía en 1993 en la denominada Cumbre de Copenhagen, constituyó un antes y un después en el desarrollo sociopolítico rumano y en las relaciones entre los países del Este saté- lites de la URSS y Bruselas. Hasta 2007, Rumanía tuvo que llevar a cabo cambios estructurales a nivel político, judicial y económico que indudablemente fueron complejos de implantar en un país que más que un proceso de europeización, vivió un proceso de euro-balcanización (Gallagher, 2009). Rumanía asimiló leyes, valores y los procedimientos de toma de decisiones europeas, pero singularmente a un nivel superficial (Marcu, 2011). A partir del año 2007, cuando finalmente Rumanía entra en la UE, han tenido lugar transformaciones significativas de adaptación para alcanzar los estándares europeos establecidos. El proceso de transición resultó en una crisis económica sin precedentes que incidió en un incremento importante de los niveles de pobreza, siendo uno de los colectivos más perjudicados el de etnia gitana (Gutiérrez, 2017), el cual constituía hogares numerosos, baja formación y, además, vivían en el hábitat rural, mermando seriamente las condiciones de vida por la complejidad en el acceso a determinados bienes y servicios.

La movilidad iniciada por cuantiosos rumanos, parten (en gran medida) de los procesos de integración europea que se produjeron. Estos hechos han desarrollado en la población gitana proyectos familiares o individuales de corte laboral (Griffin, 2008; Viruela y Torres, 2017). En este sentido, en este artículo se muestra en primer lugar una breve revisión de aspectos generales sobre el colectivo Rrom procedente de Rumanía, para posteriormente analizar el estudio de caso llevado a cabo en el asentamiento chabolista de El Gallinero (Madrid) y el Área Metropolitana de Barcelona. Se analizan diversos aspectos en cuanto a la intervención social por medio de proyectos de Trabajo Social para la inclusión social. Se considera este análisis como ejemplo para desarrollar intervenciones que puedan ayudar a implantar proyectos sociales con colectivos vulnerables y que guarden similitud tanto por origen como por sus necesidades.

\section{Movilidad Rrom y exclusión social}

En numerosas ocasiones cuando se habla del Pueblo Gitano, "el discurso se centra en el tratamiento de los mismos como sociedad tradicional, donde el nomadismo ha sido el principio que regía el modelo pasado o presente" (Gutiérrez, 2017:43). Actualmente no todos los gitanos son nómadas, pero su carácter itinerante ha sido, sin embargo, una de las notables características 
que los han distinguido de los sedentarios, ya sea en la realidad o en el campo de lo imaginario (Berthier, 1979).

En Rumanía, los gitanos fueron asociados automáticamente con las difíciles situaciones que atravesaba el país, es decir, formaban parte de una identificación colectiva negativa, sobre todo, por la interpretación que establecía que los gitanos no eran partidarios del proyecto socialista durante la época dictatorial de $\mathrm{Ni}$ colae Ceaucescu (1967-1989). Muchos de ellos ante el panorama excluyente y de dificultad económica, viajaron a lo largo de los años 90 a países como Alemania, Austria o Francia, sin embargo, y como sostiene Marcu (2013), los obstáculos a la inmigración impuestos por estos países hicieron que la movilidad se orientara hacia los países de la cuenca mediterránea (España, Italia o Portugal). Siguiendo a Crowe (2003), los movimientos de esta población podrían considerarse una estrategia de supervivencia que se desarrolla bajo las distintas circunstancias vividas a lo largo del tiempo.

Elrick y Ciobanu (2009) y Marcu (2013) señalan cómo en la emigración rumana hacia España, los investigadores señalan tres etapas diferenciadas (Pajares, 2007; Reher et al, 2008; Viruela, 2006). La primera de ellas, comprendida entre 1990-1995, cuando se registraron 325.999 solicitudes de asilo político en Europa Occidental (Diminescu, 2003). Según las estadísticas del año 2006, entre 1990 y 1995, España atrajo un número muy reducido de rumanos, sin embargo, a partir de 1996, las rutas internacionales cambiaron de dirección, dirigiéndose hacia países del sur, siendo Italia y España los principales destinos. El periodo 2002-2007, estuvo marcado por la apertura fronteriza mediante el Tratado de Schengen que supuso la libre circulación de personas en el territorio comunitario. Esta etapa se configura como una de las más importantes en la emigración rumana hacia España. El último periodo comienza en el año 2007 y está marcado por el ingreso de Rumanía en la UE. En solo un año, los flujos aumentaron desde 211.325 a 603.889 personas, convirtiéndose los rumanos en ciudadanos europeos con libre derecho de circulación (Favell, 2008 en Marcu, 2013).

Las migraciones son en sí mismas, procesos de movilidad social que albergan un proyecto transnacional por el cual los sujetos insertos en ella pretenden mejorar la calidad de vida individual, grupal o familiar (Aysa-Lastra y Cachón, 2013; Gutiérrez, 2017a; Suárez-Grimalt, 2017). Según Viruela (2016) tanto en geografía como en sociología, el término de movilidad se emplea esencialmente para referirse al desplazamiento de personas en el espacio $y$, además, ha conocido conceptualizaciones y distintos campos de aplicación (Imbert et al, 2014).

En este caso la movilidad incorpora elementos que guardan relación con la libertad y como sostiene Bauman (2000:21) “liberarse, al hablar de emancipación, significa deshacerse de las ataduras que impiden o debilitan el movimiento, es sin más, sentirse libre en cuanto a la capacidad de actuar y por supuesto de moverse". Por tanto, la movilidad concentra elementos de la teoría migratoria conectados en la combinación de conceptos como frontera, transnacionalismo o lugar (Anderson y O'Dowd, 1999; Gustafson, 2009; Marcu, 2013; Vertovec, 2001).

En la actualidad, y a pesar de llevar poco más de una década incorporada Rumanía en la Unión Europea, es posible detectar comunidades romaníes asentadas en las periferias de grandes ciudades europeas en condiciones de marginalidad y exclusión social, como son los casos de Burdeos en Francia (Gutiérrez, 2017a), Turín en Italia (Vergnano, 2014) e incluso en España, donde podemos encontrar el poblado chabolista de El Gallinero en Madrid (Gutiérrez, 2015) y en el área metropolitana de la ciudad de Barcelona (López y Aharchi, 2012). En todos los casos, salvo por características propias en cuanto a regulaciones y normas de cada país o Comunidad Autónoma (en el caso de España) los asentamientos presentan aspectos similares propios de barrios desfavorecidos donde ocupan territorios privados, fincas, edificios, casas de labranza o bien las administraciones locales ceden espacios con carácter temporal.

A lo largo de la línea histórica que se dibuja en este apartado, es relevante destacar como la carga de marginalidad iniciada en origen y que asumen los romaníes, es transportada durante toda la movilidad a lo largo del campo transnacional (Gutiérrez y Gimeno, 2019). Dicha "carga" se caracteriza (entre otras razones) por; mantener la situación de desempleo y estar inmersos en trabajos eventuales y propios de la economía sumergida (Gutiérrez, 2017a); mendicidad, sobre todo llevada a cabo por mujeres; en según qué casos y en clases más desfavorecidas continuidad de las prácticas de trabajo sexual (López-Riopedre, 2017); y por último, el Pueblo Gitano ha demostrado a lo largo de la historia la posibilidad de mantener una identidad que no tiene por qué tener vinculación territorial. Habermas (2000: 238) indica cómo "se puede compaginar una identidad cultural determinada con la pertenencia a distintas realidades estatales o nacionales". Los gitanos ya han experimentado diversos procesos desde sus principios, por un lado han vivido la práctica de la movilidad y por otro el sentir y formar parte de lugares distintos pero con el reconocimiento de todo un pueblo a nivel global.

\subsection{Periferia y barrios desfavorecidos}

Hablar de periferia y barrios desfavorecidos implica adoptar una óptica holística en cuanto a la infinidad de aspectos y procesos que suceden en dichos espacios. Teniendo en cuenta el apartado anterior en relación al proceso de movilidad de los gitanos rumanos y la creación de barrios desfavorecidos en las periferias de grandes ciudades europeas, es pertinente analizar las características que estructuran determinados entornos urbanos y aqueIlos territorios que por causas múltiples han tendido a la degradación y exclusión social. En este sentido, cada sociedad produce un espacio, el cual engloba la diversidad y realidades particulares (Lefebvre, 2013), es decir, un determinado espacio ubicado en una ciudad, si no es siempre análogo a la sociedad, es al menos el hábitat donde las sociedades progresan (Park, 2013). En dicho hábitat, hallamos diversas perspectivas y formas de aproximación o de relación entre los individuos. Como sostienen Garrido y Jaraíz (2017), en la sociedad actual se produce un incremento de la desigualdad debido mayormente a una acelerada globalización económica liberal que da lugar a una serie de cambios sociales donde se destacan, los movimientos migratorios, cambios demo- 
gráficos e incluso precarización del empleo. Por lo tanto, ante los retos que supone afrontar una crisis económica que sobre todo ha afectado al Sur de Europa, aparecen nuevas realidades y procesos de degradación de las periferias urbanas (Jameson, 1991; Sassen, 2010).

La ciudad es vista como una red urbana de intereses y relaciones articuladas y jerarquizadas (Garrido y Jaraíz, 2017), dónde sus habitantes alcanzan sus rentas a través de una actividad manufacturada multilateral que cubren sus necesidades fundamentalmente de forma local (García, 2005), gracias a una actividad regular de reciprocidad de bienes. Cada ciudad se origina a partir de la confluencia de diferentes factores de desarrollo que, como señala Fernández (2017:81), "es una confluencia provocada históricamente por fuerzas centrífugas, inercias y sinergias, resultado de la intervención de diferentes agentes que reproducen relaciones sociales asimétricas en el espacio".

Llegado a este punto y dentro del análisis de la ciudad como entorno urbano donde se manifiestan diversos procesos, cabe abordar la cuestión de barrio desfavorecido. Dentro de la interpretación y valoración de determinados territorios, hay que señalar que no todos los asentamientos o espacios cumplen igualitariamente un ciclo universal de desarrollo, sino que cada lugar en sí evoluciona según los propios individuos que los componen y las intervenciones públicas llevadas a cabo a lo largo del tiempo. Actualmente y como señala Rojo (2010), está siendo reclamada por los investigadores sociales, la investigación sobre el impacto del territorio en los comportamientos sociales, y sobre las políticas cohesivas de intervención social desde el territorio ante los problemas de marginación y exclusión social. Bajo el paradigma de que solo existe marginación si existe un poder establecido que delimite las fronteras de la tolerancia social, Rodríguez (1986) expone como la valoración de la marginalidad se entiende como una realidad producto de la estructura social, de las relaciones sociales que producen los miembros de la sociedad.

Garrido y Jaraíz (2017) partiendo de la interpretación de Subirats (2006), sostienen como en los barrios desfavorecidos se producen dos fenómenos interrelacionados; por un lado un flujo de población creciente y vulnerable (inmigrantes, minorías, desempleo, etc.) y por otro lado un flujo de salida de población que busca o logra una mejor calidad de vida. Continuando con Rojo (2010) y el impacto de determinados territorios sobre grupos o colectivos, es preciso aproximarse a su vez al concepto de barrio o territorio "sensible" que aporta la Sociología de origen francés de los años 80 incluyendo un análisis conceptual y fundamental en el propio análisis sociológico donde se incorpora además la violencia juvenil urbana. El atributo de "sensible" suma el tradicional término de "barrio desfavorecido" comúnmente utilizado en España (Rojo, 2010). Ambas interpretaciones de barrio (desfavorecido o sensible) no son excluyentes una de la otra.

A estos aspectos hay que añadir las condiciones específicas que se pueden observar en cada territorio. Con carácter general, Torres (2011) apunta características compartidas en entornos desfavorecidos o bien en asentamientos; la accesibilidad, la adecuación de las características de la vivienda o alojamiento en razón de sus necesidades y caracteres demográficos de sus ocupantes, el riesgo o la seguridad de poder disfrutar de un uso estable y continuado de la vivienda, y la propia habitabilidad (situación física y arquitectónica).
Además de las peculiaridades del contexto donde habita una población determinada, existen otros factores de vulnerabilidad y que igualmente afectan a las condiciones de vida de la población. En este sentido, se determinan múltiples factores de riesgo que alteran diaria y negativamente a las personas (Herrera, 1999). Sánchez-Teruel (2012) recoge aquellos tipos de factores de riesgo a los que se puede enfrentar un individuo;

- por un lado, aquellos factores de riesgo de carácter psicológico (como baja autoestima, escasas habilidades de afrontamiento, ansiedad, impulsividad, baja tolerancia a la frustración, rigidez cognitiva y locus de control interno;

- de carácter familiar (inestabilidad, familias no funcionales, estilos parentales discrepantes o inadecuados y modelos inapropiados;

- de tipo social (entorno conflictivo, exposición de modelos antisociales, acceso a sustancias tóxicas y escasas redes de apoyo social);

- y otros factores como el desempleo, la inactividad o el absentismo escolar en el caso de niños y niñas.

\section{Objetivo y metodología}

Este trabajo adopta un enfoque cualitativo con el objetivo de analizar la movilidad de las familias gitanas rumanas asentadas en la periferia de la ciudad de Madrid y el área metropolitana de Barcelona, así como las condiciones de vida en entornos catalogados como de riesgo o vulnerabilidad.

La investigación se desarrolla en dos periodos concretos. El primero de ellos entre 2010 y 2017 bajo la investigación doctoral del autor y el segundo de ellos corresponde entre 2017 y 2019.

En el primer periodo de investigación, se llevó a cabo un trabajo de campo de un año de duración en el asentamiento chabolista de El Gallinero (Madrid), donde se emplearon las técnicas de observación participante y entrevista. La observación fue posible por medio de la inclusión en los proyectos de intervención que la Fundación Imaginario Social desarrollaba en la zona, desde la cual se puede resaltar la inclusión por parte del investigador en actividades deportivas, de distensión, formativas y de trabajo de habilidades sociales con las familias del lugar. Hasta 2017 el número de familias asentadas en El Gallinero era de 90 aproximadamente, donde el total de la población era de 400 personas (siendo el 50\% menores de edad).

Las entrevistas, que fueron un total de 86 , eran semiestructuradas en profundidad. Se realizaron a hombres y mujeres entre 18 y 41 años (un $82 \%$ y $18 \%$ respectivamente). En El Gallinero (Madrid) se realizaron un total de 70 entrevistas y en el área metropolitana de Barcelona (en la fase posterior), 16 del total.

Las entrevistas así como la observación participante tenían como objetivo estudiar las intervenciones sociales en las que los sujetos participaban y además, analizar la movilidad y los procesos de exclusión de exclusión teniendo en cuenta cómo afecta 
el territorio a las condiciones de vida de la población. Las entrevistas tenían una duración de aproximadamente 45 minutos y fueron llevadas a cabo en los propios asentamientos, partiendo de la búsqueda de un lugar cómodo para responder a diversas cuestiones que giraban en torno a su situación social, causas y consecuencias de su movilidad hacia España. Para poder llevar a cabo las entrevistas fue necesario un periodo de adaptación y aproximación a los asentamientos debido a la desconfianza previa por parte de los sujetos hacia el investigador. Por lo tanto fue necesario crear la confianza suficiente para poder desarrollar la investigación (Kholi, 2006; Gimeno y Gutiérrez, 2019).

El segundo periodo de la investigación (2017-2019) contempló la realización de entrevistas y la investigación bibliográfica y documental sobre el colectivo Rrom en el área metropolitana de Barcelona (en concreto en el barrio de Poblenou), donde fue posible además realizar una aproximación a diversos asentamientos y observar similitudes con la población asentada en Madrid. Por otro lado, en septiembre de 2018 se produjo el desalojo de El Gallinero, por lo que se hizo hincapié en observar los medios empleados por parte de las Administraciones (Locales y Regionales) en el desmantelamiento del lugar.

Por último, la información obtenida en la trascripción de las entrevistas, fue ordenada por categorías de análisis (movilidad, juventud, estrategias, familia, exclusión social y empleo) por medio de Excel 2007 para identificar unidades de significado aproximado. Luego se codificaron "horizontalmente (el conjunto de entrevistas), generando categorías analíticas más amplias y palabras clave (Pastoor, 2015). Posteriormente, se integró la información relacionando las categorías entre sí con los fundamentos teóricos de la investigación.

\section{Resultados}

4.1. Origen y movilidad: condiciones de vida en los asentamientos

Pese a las existentes inversiones públicas de carácter social a nivel local en materia de erradicación de barrios desfavorecidos, en la actualidad continúan desarrollándose núcleos chabolistas en grandes ciudades cómo es el caso de Madrid y Barcelona. En ambos territorios es observable que su origen (cuando se habla de familias o comunidades Rrom) viene dado previamente por el tránsito por varios asentamientos de similares características, ya sea en España o bien de otros países de la Unión Europea (hay que matizar que también se dan casos en los que las familias vienen a España directamente desde Rumanía). En los casos estudiados es destacable cómo diversas unidades familiares han ido transcurriendo por diversos asentamientos desarrollando comunidades, las cuales han ido padeciendo desalojos, desahucios o realojos y por tanto contribuyendo de algún modo a la movilidad del colectivo dentro de la geografía española. En el caso de El Gallinero, el cual data de 2001, es fruto del derribo de otras unidades de realojamiento especial como La Quinta, La Celsa, La Rosilla y las Barranquillas (Gutiérrez, 2015). Además, el desalojo del Barrio de Malmea en 1999 y el intento fallido de la administración local por crear "Campamentos" específicos, donde atender a esta población, perturbaron aún más la situación provocando la peregrinación a lugares alternativos, hasta llegar a asentarse en el Poblado de El Gallinero. En el caso catalán el proceso es similar, se observa cómo diversas comunidades se han desarrollado por la movilidad entre diversas ciudades del área metropolitana de Barcelona, como es el caso de Santa Coloma de Gramenet y Badalona.

En este sentido cabe indicar de nuevo el proceso de desalojo que padeció El Gallinero en septiembre de 2018. Al ser reciente todavía dicho proceso, es pertinente observar la movilidad de la población y su posibilidad de dispersión por otras áreas del espacio madrileño e incluso español.

No tenemos a dónde ir, no tenemos dinero para nada. Solo queremos un lugar seguro para nuestros hijos. Hombre, 35 años. Madrid.

En ambos casos observados, las comunidades asentadas viven en condiciones precarias en cuanto a la calidad de las construcciones. Chapas, plásticos y maderas sirven para "levantar" chabolas de aproximadamente $15-20 \mathrm{~m}^{2}$ en solares o espacios que son de titularidad privada. La luz y el agua son obtenidas por medio de enganches ilegales a las redes públicas. En el caso de El Gallinero y a lo largo de los años han habido varios incendios provocados por el mal uso de cables y contadores, lo cual ha provocado la rápida propagación del fuego por las chabolas con todos los riesgos que contraen (posibles pérdidas humanas, quemaduras, pérdida de enseres y documentación como pasaportes y documentos de identidad nacional). Por otro lado, las administraciones públicas (en ambos escenarios) no han suministrado materiales de construcción a la población con el fin de mejorar las condiciones de vida (ya sea con letrinas, maderas para la construcción de chabolas o asfaltado del terreno). Es observable la proliferación de roedores e insectos provocados, sobre todo, por la no canalización de aguas y limpieza de los asentamientos. En el caso madrileño hay que añadir la proximidad con el vertedero de Valdemingómez, el cual agrava la situación de degradación del entorno por el hedor y el continuo tránsito de camiones de limpieza y recogida de basuras.

Siempre huele mal y hay muchas ratas. A veces entran en casa para robar comida y han mordido a alguna vez a mi marido. Mujer, 23 años. Madrid.

Aquí existe un problema de basuras. Los bidones salieron ardiendo y solo nos queda dejarlo todo en la calle tirado. Hombre, 27 años. Barcelona.

Las prácticas de subsistencia que los Rrom desarrollan en ambos asentamientos coinciden en lo siguiente: 
- Venta de diversos útiles: comercio de pequeños enseres como ropa, zapatos y madera a sus vecinos. En relación al calzado y vestimenta, éstas suelen ser recogidas y almacenadas, para a la postre venderlas cuando alguien nuevo llega al asentamiento, nace un bebé o bien cualquier persona requiere obtener ropa nueva.

- Alquiler: como parte del proceso de movilidad, cuando una familia tiene que trasladarse a otro lugar por un periodo de tiempo, alquila su casa a otras familias con el propósito de no perderla y adquirir dinero extra. Las primeras familias que originaron al asentamiento, suelen ser las que alquilan sus casas a otros. Algunas casas son de propiedad de una familia. Hay que indicar que en caso barcelonés, muchas familias ocupan edificios cuando tienen la posibilidad, sin embargo, el contexto comunitario brinda mayor protección y seguridad ante intentos de desalojos y desahucios, por lo que muchas familias optan por la ocupación de solares para posteriormente construir sus viviendas.

- Préstamos: al carecer las familias de cuentas corrientes en bancos con las que transferirse dinero, es usual que se presten dinero pero con altos intereses, ya que según la propia población, existen ciertos riesgos que el dinero no les sea devuelto. Este medio es común, conociéndose casos de mayor empobrecimiento por no poder abonar al prestamista el dinero acordado.

- Trabajos relacionados con economía sumergida y baja cualificación profesional: Se realizan trabajos de recogida de chatarra, cartones o aquellos objetos de los que pueden obtener algún beneficio, los cuales posteriormente son vendidos a plantas de reciclaje. Trabajos de transporte de materiales de construcción es otra de las labores observadas y, en la mayoría de los casos, suele llevarse a cabo por hombres.

- Mendicidad: por lo general son las mujeres las que acuden al centro de las ciudades con el propósito de mendigar, sobre todo en puertas de iglesias o supermercados. No acumulan amplias cantidades de dinero pero este sustento resulta de vital importancia para la unidad familia. Las niñas en edades más avanzadas para la comunidad (normalmente entre 13 y 14 años) comienzan a su vez a ayudar a sus madres en esta tarea.

- Ayuda de ONGs: son diversas las ONG que ayudan a la población con bienes materiales y con equipos de profesionales especializados. Según el tiempo que lleve el asentamiento y las necesidades propias de la población, son observables distintas ayudas, las cuales sobre todo consisten en ayudas materiales, formativas, de asesoría jurídica, sanitarias, deportivas y de distensión.

\subsection{Exclusión social y desadaptación}

A pesar de la tendencia que desarrollan los Rrom de vivir en comunidades y cómo estas pueden ayudar a la unión colectiva e inclusión social, la participación política o asociativa no es visible en los contextos donde se ha realizado la investigación. Se observa en primer lugar cómo la propia organización social interna de las comunidades contiene déficits. La desconfianza, sobre todo manifestada con aquellas personas nuevas que por medio de la movilidad constante llegan al asentamiento, juega un papel importante ya que no permite en determinados casos que se afiancen lazos de unión y reciprocidad entre vecinos. Aunque no está establecida una estructura social donde cada persona se tiene que ocupar de determinadas tareas en pro de la comunidad, si existen personas que tienen cierto papel jerárquico y que asumen en numerosas ocasiones el papel de interlocutor con entidades sociales y administraciones. Estas relaciones pueden tener dos niveles, por un lado de control de acceso a la comunidad y, por otro, el poseer un papel importante en la comunidad puede hacer que se obtengan más beneficios o prestaciones sociales (ayudas por ejemplo en alimentos y vestimenta) que ayuden específicamente a la propia unidad familiar (posición privilegiada).

El conocimiento recíproco entre las familias en los asentamientos tiene un papel relevante para mantener una atmósfera de orden y capacidad de reacción ante cualquier amenaza (redadas o desalojos). Se puede observar cómo a pesar de haber cierto orden en cada comunidad, la exclusión social interna en determinados casos, Ileva a un cierto aislamiento en las casas. Estos casos se posibilitan sobre todo cuando existen familias con diferentes estatus económicos, cuando hay personas que tienen relación con grupos criminales, o bien hay cierta desconfianza con los recién llegados.

Mucha gente ha venido en los últimos años y no me gustan. Roban y hacen cosas malas. Yo no quiero eso para mi familia. Hombre, 33 años. Madrid.

A este clima de exclusión interna en las comunidades se le añade la exclusión social por parte de la sociedad general hacia el desarrollo y proliferación de espacios desfavorecidos como los que tenemos en cuenta en este trabajo. Las relaciones de convivencia son escasas y en numerosas ocasiones se limitan a los momentos de interacción que se establecen cuando se llevan prácticas de mendicidad en puertas de supermercados o iglesias. En el caso concreto de niños y niñas que están escolarizados en colegios o institutos, las relaciones aumentan sobre todo cuando los padres se preocupan por la educación de sus hijos, participando en actividades extraescolares o tutorías. Sin embargo, dichas relaciones son más bien deficitarias debido al absentismo escolar existente, lo cual no solo incide negativamente en los menores sino también de algún modo impide que se mantengan en el tiempo relaciones de convivencia que pueden ayudar a la inclusión social del colectivo.

Por último, es destacado detallar la aparición de procesos de aculturación como tácticas de adaptación social. En el caso de los Rrom y en concreto de las mujeres que utilizan faldas largas y en según qué casos pañuelos que cubren la cabeza (elementos muy característicos de este colectivo), la posibilidad de encontrar un empleo o simplemente no quedar excluida por su condición de gitana y rumana, hace que se desarrollen procesos de aculturación donde las mujeres tienen que cambiar su forma de vestir por aquellas que podemos categorizar como occidentalizadas. Este ejemplo de aculturación choca drásticamente con el mantenimiento de la propia cultura, dónde sobre todo, las personas de 
mayor edad ejercen presión social sobre aquellas que pretenden simplemente encontrar un trabajo o no ser tan discriminadas.

\subsection{Etapas de desarrollo}

La investigación empírica en los asentamientos en Madrid y Barcelona nos muestran cómo en el desarrollo psíquico y físico de niños y niñas es pertinente observar los procesos de madurez que transcurren. Se observan dos etapas diferencias y comunes que tienen sobre todo un origen compartido, las tácticas empleadas por salir de la pobreza y la exclusión social (Gutiérrez, 2015).

La primera de las etapas y que es denominada cómo Familiar-escolar, se desarrolla entre el nacimiento y los trece años de edad (aproximadamente). Es este estadio los niños y niñas siguen acudiendo a la escuela, no existe presión social por querer que los menores no estudien y se dediquen a otras labores en los asentamientos, y existe una preocupación y protección comunitaria ante aquellos más vulnerables. A los trece años se observan cambios relevantes en el comportamiento, por un lado en las chicas es el momento en que la madurez sexual se manifiesta y por tanto se comienza con el casamiento. Por otro lado, los chicos poco a poco acompañan a sus padres en las tareas de recogida de chatarra y por tanto, en ambos casos (chicos y chicas), los niveles de absentismo escolar aumentan y se desarrolla la segunda etapa denominada como de Proyección a la vida adulta.

La situación económica y social es un aspecto clave en el salto de una etapa a otra. Si la familia muestra bajos niveles económicos será más probable que fomenten entre sus hijos la idea de que han de trabajar a edades tempranas, como forma de superar y llegar a obtener un determinado nivel que les permita combatir los problemas que tienen como origen la pobreza y la propia exclusión social. La influencia del entorno es un factor distinguido en esta etapa. La percepción de la comunidad (tanto Madrid como Barcelona) en cuanto que los jóvenes a una determinada edad dejan de algún modo de ser niños para convertirse en hombres y mujeres, acentúa aún más el problema que deriva de esta ciclo. Los niños y niñas tienen que asimilar y aceptar el lugar donde viven, y con ello, saber desenvolverse lo mejor posible para superar los problemas resultantes de la pobreza (pasar hambre, sentir rechazo, padecer falta de higiene, tener poco acceso a medicamentos, etc.).

A su vez existen otros aspectos que inciden en dichas etapas o en este tránsito prematuro entre la niñez y la vida adulta, como son: problemas derivados de la ocupación del terreno ilegalmente (se desarrollan redadas y desalojos forzosos); problemas por las condiciones del asentamiento (por lo cual los niños y niñas se ocupan en gran medida del cuidado de hermanos mientras que los padres buscan soluciones a los problemas de abastecimiento como luz, agua y comida); las restricciones de las entidades sociales que operan en el lugar por limitación de actividades a áreas específicas como educativas y distensión (con poco apoyo de la administración local y regional); y carencia de planes específicos de atención a población excluida donde se desarrollen proyectos de intervención social para la inclusión y fomento del empleo.

\subsection{Proyectos de intervención social}

El nivel de ayudas enmarcadas dentro de las intervenciones sociales de entidades privadas con carácter social, puede mejorar en cierta forma la calidad de vida de las personas asentadas en entornos catalogados como desfavorecidos. En el caso madrileño, eran varias las organizaciones sociales que trabajaban en el asentamiento desde diferentes áreas (vivienda, salud y educación). En el caso barcelonés el número de entidades era menor incidiendo sobre todo en vivienda y educación. Al hablar de viviendas se hace referencia a aquellos proyectos orientados a mejorar las estructuras de los hogares ya sea en su mantenimiento como con ayudas en especies; en relación a salud, aquellas intervenciones dirigidas a la atención primaria y de orientación; y en relación a educación, aquellos proyectos dirigidos a la prevención del absentismo escolar, refuerzo educativo y relaciones entre la comunidad y las instituciones educacionales como colegios e institutos.

En ambos asentamientos era vital para las personas contar con el apoyo de organizaciones no gubernamentales. En ciertas ocasiones se llevaban a cabo denuncias por abuso policial; denuncias por realizar redadas nocturnas por como afectan éstas al desarrollo de los niños; búsqueda de mejoras en materiales de construcción y adecentamiento de los terrenos; y sobre todo se establecían puentes de unión entre sociedades que servían para integrar a la población y mejorar su situación.

Dentro del contexto de crisis económica que todavía afecta a Europa, es relevante indicar como los recortes producidos en los últimos años en materia de educación, sanidad, inclusión social y fomento del empleo, han afectado a las organizaciones, las cuales han visto reducidas sus partidas económicas y por tanto recortando la atención profesional y la calidad de las intervenciones por no contar con los medios necesarios.

En este estudio en total se han analizado, en ambos asentamientos, un total de 7 proyectos de intervención en las áreas anteriormente citadas, de los cuales se extrae la importancia de asegurar su permanencia en el tiempo; tener profesionales formados en diversas áreas ante respuestas rápidas de actuación (redadas, desalojos, emergencias sanitarias); incorporar a la propia población en el desarrollo de los proyectos para asegurar su estabilidad y apoyo; y desarrollar redes entre diversas instituciones como medio para no hacer duplicidades y a su vez tener una "voz" común en la relación con las administraciones públicas.

\section{Conclusiones}

Este artículo ha comparado asentamientos chabolistas, en las ciudades de Madrid y Barcelona, donde vive población rumana de etnia gitana, analizando las condiciones de habitabilidad, medios de subsistencia y la incidencia negativa que tienen entornos 
como los abordados sobre la población que en ellos se asienta. El artículo ha venido a la conclusión de que las autoridades locales intervienen de manera diferente ante problemas comunes y cómo la población a pesar de vivir en espacios diversos, desarrollan similares procesos de movilidad y habitabilidad los cuáles en cierta medida, afectan a niños y niñas negativamente por los continuos desplazamientos que a nivel familiar se producen y además, por la presión que padecen desde las administraciones públicas (locales y regionales) por querer establecer prácticas de control y reasentamiento en otros espacios por la peculiaridad del colectivo en ocupar territorios o lugares de carácter privado.

Los flujos migratorios, el decaído mercado laboral, la crisis económica, la exclusión social y la pobreza han desencadenado procesos de movilidad que han transformado a las sociedades receptoras en algunas ciudades españolas. La tendencia a la concentración de población con cierta debilidad en las economías familiares, escasez y/o ausencia de servicios básicos de carácter comunitario en las periferias de ciudades cómo Madrid y Barcelona, es hoy día uno de los grandes retos a tener en cuenta por parte de las administraciones públicas (en sus distintos niveles) así cómo por las entidades sociales debido, mayormente, por su complejidad y capacidad de respuesta ante situaciones de exclusión social que les exigen estar preparadas ante situaciones de emergencia social.

El presente estudio revela la necesidad de armonizar los protocolos de atención social a la población Rrom debido, sobretodo, por su movilidad continua por la Unión Europea desarrollando prácticas similares de subsistencia y que además comparten procesos de exclusión social y pobreza desde origen. Por lo tanto es pertinente en primer lugar ofrecer información y orientación a las familias sobre su situación administrativa y social (teniendo en cuenta que son europeos) pero además escuchando las necesidades manifestadas que permitan una mayor integración social tanto en los proyectos que se implanten como en la sociedad en general.

En este artículo se resalta la importancia de que la población Rrom desarrolle acciones asociativas y activismo político como medio para obtener mayor visibilidad ante los problemas sociales que padecen también como ciudadanos en igualdad de condiciones. Además, y fruto de la escasa participación social del colectivo, se observa cómo se manifiestan procesos de aculturación en la población más joven, los cuales sufren resistencias desde las propias comunidades debido mayormente por el miedo a la pérdida de identidad cultural que los define. Es decir, los procesos existentes de inclusión social o aquellos potencialmente implantables, en ocasiones chocan con las resistencias de la propia población, la cual más allá de no querer estar incluida en la sociedad, alberga reticencias por no desear perder su propia identidad y costumbres lo cual va en detrimento de organizar e implantar redes asociativas. Dicho esto, hay que tener en cuenta que la constante movilidad del colectivo no permite la estabilidad en la implantación de medidas comunitarias o asociativas, por lo que aunque éstas se desarrollaran no tendrían toda la estabilidad necesaria en un comienzo para que se mantuvieran en el tiempo.

Este trabajo incluye además la distinción de etapas de desarrollo que se manifiestan en niños y niñas en torno a los 13 años de edad. Sería interesante analizar en profundidad cómo se manifiestan dichas etapas en entornos o contextos de riesgo como los observados, como medio para conocer vías que permitan una reducción del absentismo escolar y que los menores sigan realizando actividades acordes con su edad.

Finalmente, este artículo sugiere que los Estados europeos deberían proporcionar una atención inmediata y holística a las familias en tránsito. Este hecho ofrecería una atención acorde a las necesidades propias de las familias y se podría reducir el número de asentamientos por un acceso alternativo a viviendas sociales y de gestión pública, de tal forma que ayuden a reducir las condiciones de pobreza y exclusión, ofreciendo a su vez programas educativos y sanitarios. 
ALGUACIL, J. (2006): "Barrios desfavorecidos: diagnóstico de la situación española", en F. Vidal, (dir.), V Informe FUHEM de políticas sociales: La exclusión social y el Estado de bienestar en España, Madrid, Fundación Hogar del empleado.

ANDERSON, J. y O'DOWD, L. (1999): "Borders, border regions and territoriality: contradictory meanings, changing significance", Regional Studies, 33, pp. 593-604.

AYSA-LASTRA, M. y CACHÓN, L. (2013): "Movilidad ocupacional segmentada: el caso de los inmigrantes no comunitarios en España", REIS. Revista Española de Investigaciones Sociológicas, 144, pp. 23-47. https:// doi.org/10.5477/cis/reis.144.23

BAUMAN, Z. (2000): Modernidad líquida, Argentina, Fondo de Cultura Económica.

BERTHIER, J. C. (1979): "La socialización del niño gitano", Revista Internacional de Ciencias Sociales, XXXI(3), pp. 409-426.

CLAVE-MERCIER, A. y OLIVERA, M. (2018): "Inclusion and the 'Arts of Resistance.' How Do Roma Migrants Develop Autonomy in the Context of Inclusion Policies? Intersections", East European Journal of Society and Politics, 4(2), pp. 149-168.

CROWE, D. (2003): "The International and Historial Dimensions of Gitanosni Migration in Central and Eastern Europe", Nationalities Papers, 31(1), pp. 81-94.

DELL'AGNESE E. y VITALE T. (2007): "Rom e sinti: una galassia di minoranze senza territorio", en G. Amiotti y A. Rosina (eds.), Tra identità ed integrazione: passato e presente delle minoranze nell'Europa mediterranea, Milán: Franco Angeli.

DIMINESCU, D. (2003): "Introduction", en D. Diminescu, (ed.), Visibles Mais Peu Nombreux. Les Circulations Migratoires Roumaines, Paris, Editions de la Maison des sciences de l'homme, pp. 1-23.

ELRICK, T. y CIOBANU, O. (2009): "Migration networks and policy impacts: Insights from Romanian-Spanish migrations", Global Networks, 9, pp. 100-116.

FAVELL, A. (2008): "The new face of East-West migration in Europe", Journal of Ethnic and Migration Studies, 34, pp. 701-716.

FERNÁNDEZ, S. (2017): Sociología, identidad e iconografía en la ciudad contemporánea: el caso de A Coruña. Tesis Doctoral, Universidades da Coruña.

GALLAGHER, T. (2008): Modern Romania: The End of Communism, the Failure of Democratic Reform, and the Theft of a Nation, Manchester, University Press.

GARRIDO, M. y JARAÍZ, G. (2017): "Políticas inclusivas en barrios urbanos vulnerables", Áreas. Revista Internacional de Ciencias Sociales, 36, pp. 141-151.

GIMENO, C. y GUTIÉRREZ, J. D. (2019): "Fostering unaccompanied migrating minors. A cross-border comparison", Children and Youth Services Review, 99, pp. 36-42.

GUSTAFSON, P. (2009): "Mobility and territorial belonging", Environment and Behavior, 41, pp. 490-508.

GUTIÉRREZ, J. D. (2017): "Imaginarios sociales de la itinerancia dentro de la diáspora gitana", Revista Interdisciplinaria sobre Imaginarios Sociales, Imagonautas, 9, pp. 40-57.

GUTIÉRREZ, J. D. (2017a): "Movilidad del Pueblo Gitano de Europa del Este en el asentamiento chabolista de El Gallinero", Anduli, Revista Andaluza de Ciencias Sociales, 16, pp. 73-89.

GUTIÉRREZ, J. D. (2015): “Los menores gitanos rumanos de El Gallinero: Etapas de desarrollo en un contexto de riesgo", Revista Electrónica de Investigación y Docencia (REID), 13, pp. 27-44.

GUTIÉRREZ, J.D. y GIMENO, C. (2019): "Jóvenes romaníes en contextos chabolistas: movilidad y contextos de exclusión en España y Francia", Revista de Humanidades, 38, pp. 135-160.

GRIFFIN, C. (2008): Nomads under the Westway Irish Travellers, Gypsies and other traders in west London, Hertfordshire, University of Hertfordshire Press.
HABERMAS, J. (2000): The inclusión of the other. Studies in Political Theory, Cambridge Mass, MIT Prees.

HARPER, K., Steger, T. y Filcak, R. (2009): "Environmental Justice and RomaCommunities in Central and Eastern Europe", Environmental Policy and Governance, 19, pp. 251-268.

HERIN, R. (2008): "Violencias en las periferias urbanas francesas. Los disturbios de otoño de 2005", Scripta Nova. Revista electrónica de Geografía y Ciencias Sociales, XII, 270 (96).

HERRERA, P. (1999): "Principales factores de riesgos psicológicos y sociales en el adolescente", Revista Cubana de Pediatría, 71, pp. 39-42.

IMBERT, C., Duducs, H., Dureau, F. y Giraud, M. (2014): D'une métropole á l'autre. Pratiques urbaines et circulations dans l'espace européen, París, Armand Colin.

JAMESON, F. (1991): El postmodernismo o la lógica cultural del capitalismo avanzado, Madrid, Ed. Paidós Ibérica.

JORDI SANCHEZ, M. y Aix, F. (2009): El vandalismo como fenómeno emergente en las grandes ciudades, Actual 42. Sevilla, Ed. Fundación Centro de Estudios Andaluces.

KOHLI, R. (2006): "The comfort of strangers: Social work practice with unaccompanied asylum seeking children and young people in the UK" Child and Family Social Work, 11, pp. 1-10. https://doi.org/10.1111/ j.1365-2206.2006.00393.x.

LEFEBVRE, H. (2013 [1974]): La producción del espacio, Madrid, Capitán Swing.

LÓPEZ, Ó. y AHARCHI, N. (2012): “Discursos sobre la inmigración rrom (gitana) rumana en Barcelona", Revista Discurso y Sociedad, 6 (2), pp. 543-590.

LÓPEZ-RIOPEDRE, J. (2017): "Migraciones Al Margen: grupos rumanos, diversidad y control social", Revista Internacional de Estudios Migratorios, 7 (4), pp. 229-256.

MAGAZZINI, T.y PIEMONTESE, S. (2016): "Roma migration in the UE: the case of Spain between new and old minorities", Migration Letters, 13, (2), pp. 228-241.

MARCU, S. (2013): “La movilidad transfronteriza de rumanos en España en tiempos de crisis", Revista Internacional de Sociología (RIS), 71 (1), pp. 115-141. https://doi.org/10.3989/ris.2012.01.18.

MARCU, S. (2011): "Rumanía después de su ingreso en la Unión Europea: balance desde una perspectiva global", Revista de Historia Actual, 9, (9), pp. 53-64.

PAJARES, M. (2007): Inmigrantes del Este. Procesos migratorios de los rumanos, Barcelona, Icaria

PARK, R. E (2013): "Sociología, Comunidad y Sociedad", Empiria. Revista de Metodología de Ciencias Sociales, 25, pp. 195-212.

PASTOOR, L. D. W. (2015): "The mediational role of schools in supporting psychosocialtransitions among unaccompanied young refugees upon resettlement in Norway", International Journal of Educational Development, 41, pp. 245-254. https://doi.org/10.1016/j.ijedudev.2014.10.009.

QUERALTÓ, A. (2005): Curs de Romaní, Barcelona, Lacho Baji Cali.

REHER, D. S., CORTÉS-ALCALÁ, L., GONZÁLEZ-QUIÑONES, F., REQUENA, M., SÁNCHEZ-DOMÍNGUEZ, M. I., SANZ-GIMENO, A. y STANEK. M. (2008): Informe Encuesta Nacional de Inmigrantes (ENI-2007), Madrid, Instituto Nacional de Estadística. Consultado 14 septiembre 2011 (http:// www.ine.es/inebmenu/mnu_migrac.htm\#2).

RODRÍGUEZ, Á. (1986): Transeúntes e indigentes. Estados de necesidad y respuesta social, Madrid, Concejalía de Servicios Sociales.

ROJO, T. (2010): "Violencia juvenil y territorios urbanos", Anduli, Revista Andaluza de Ciencias Sociales, 9, pp. 9-28.

SÁNCHEZ-TERUEL, D. (2012): "Factores de riesgo y protección ante la delincuencia en menores jóvenes", RES, Revista de Educación Social, 15.

SASSEN, S. (2010): Territorio, autoridad, derechos: De los ensamblajes medievales a los globales, Buenos Aires, Katz.

SUÁREZ-GRIMALT, L. (2017): “Migración y movilidad social: una aproximación desde las estrategias de acumulación de activos de la población latinoamericana en España", Revista Española de Sociología, 26 (3), pp. 2-14. https://doi.org/10.22325/fes/res.2017.27. 
SUBIRATS Humet, J. (dir.) (2006): “Es el territorio una variable significativa en los procesos de exclusión e inclusión social" en $V$ Seminario de Investigación en Ciencias Políticas, UAM-Working Paper, 65.

TORRES, F. J. (2011): "El territorio de los desheredados. Asentamientos chabolistas y experiencias recientes de erradicación en Sevilla", Hábitat y Sociedad, 3, pp. 67-90.

VASSILEV, R. (2004): "The Roma of Bulgaria: A pariah minority", The Global Review of Ethonopolitics, 3(2), pp. 40-51.

VERGNANO, C. (2014):"Conexiones entre una agresión romófoba y un proyecto de regeneración urbanística en la periferia de Turín, Italia. Una aproximación crítica", Revista del área de estudios urbanos, Quid, 4, pp. 84-109.

VERTOVEC, S. (2001): "Transnationalism and identity", Journal of Ethnic and Migration Studies, 27, pp. 573-582.

VIRUELA, R. (2016): "La movilidad interna e internacional de los inmigrantes rumanos durante la crisis", Scripta Nova, Revista Electrónica de Geografía y Ciencias Sociales, 536, pp. 1-28.

VIRUELA, R. (2006): "Inmigrantes rumanos en España. Aspectos territoriales y procesos de sustitución laboral", Scripta Nova. Revista Electrónica de Geografía y Ciencias Sociales, 10, pp. 205-228.

VIRUELA, R. y Torres, F. (2017): "La movilidad internacional de los inmigrantes ecuatorianos y rumanos durante la crisis económica en España", Studi Emigrazione, 206, pp. 215-234. 